\title{
ENAAG: Uma ideia acadêmica de sucesso na construção de um espaço de formação profissional na Universidade Federal Rural da Amazônia - UFRA (Belém - PA)
}

\author{
Bruna Cristina Nascimento de Oliveira \\ Engenheira Agrônoma pela Universidade Federal Rural da Amazônia. Participou como Coordenação das \\ edições IV, V, VI, VII, VIII, IX do Encontro Amazônico de Agrárias - ENAAG - Tem experiência na área de \\ Agronomia, com ênfase em fitopatologia. Estagiária do Instituto Sócio-Ambiental Floranativa - ISAF \\ 凶b.cristina100@hotmail.com

\section{Deyvison Andrey Medrado Gonçalves} \\ Engenheiro Agrônomo - Engenheiro Florestal - Mestre em Agronomia - Doutorando em Agronomia na \\ Universidade Federal Rural da Amazônia e atualmente em período sanduíche na Tulane University - \\ Desenvolve pesquisa nas áreas de Fertilidade e Química do Solo - Determinação de Metais Pesados \\ (Métodos USEPA) e Fitorremediação \\ $\triangle$ deyvisonmedrado@gmail.com
}

\section{Resumo:}

O evento surgiu com a proposta de preencher uma lacuna na questão de formação complementar no campus Belém da UFRA, uma vez que até então o único evento recorrente era o seminário de iniciação científica da instituição. De forma semelhante com modelo já desenvolvido pelos acadêmicos dos cursos de Agronomia em Altamira (UFPa), Marabá (UFPa) e Imperatriz-MA (UEMa). O ENAAG surgiu para ser um evento pensado, executado e direcionado para os estudantes e para a comunidade envolvida com os temas a serem abordados. No entanto, a necessidade do desenvolvimento de habilidades para a idealização e realização do evento acabou se tornando um espaço de formação/aperfeiçoamento profissional para os discentes envolvidos.

Palavras-chave: Formação Acadêmica, Ciências Agrárias, Aperfeiçoamento Profissional.

\section{ENAAG: An Academic Idea of Success in building a professional training at the Federal Rural University of Amazonia - UFRA (Belém - PA)}

\begin{abstract}
:
The event came up with the proposal to fill a gap in the issue of complementary training at the Belem campus of UFRA, since until then the only recurring event was the institution's scientific initiation seminar. In a similar way with a model already developed by the academics of the courses of Agronomy in Altamira (UFPa), Maraba (UFPa) and Imperatriz-MA (Uema). ENAAG has emerged to be an event designed, executed and directed to the students and the community involved with the topics to be addressed. However, the need to develop skills for the idealization and realization of the event turned out to be a space of professional training / improvement for the students involved.

Keywords: Academic training, Agrarian Sciences, Professional Development.
\end{abstract}




\section{ENAAG: Una idea académica de éxito en la construcción de un proyecto espacio de formación profesional en la Universidad Federal Rural de la Amazonia- UFRA (Belém - PA)}

\section{Resumen:}

El evento surgió con la propuesta de llenar una laguna en la cuestión de formación complementaria en el campus Belem de la UFRA, ya que hasta entonces el único evento recurrente era el seminario de iniciación científica de la institución. De forma similar con modelo ya desarrollado por los académicos de los cursos de Agronomía en Altamira (UFPa), Maraba (UFPa) y Emperatriz-MA (UEMa). El ENAAG surgió para ser un evento pensado, ejecutado y dirigido para los estudiantes y para la comunidad involucrada con los temas a ser abordados. Sin embargo, la necesidad del desarrollo de habilidades para la idealización y realización del evento acabó convirtiéndose en un espacio de formación / perfeccionamiento profesional para los alumnos involucrados.

Palabras clave: Formación académica, Ciencias Agrarias, Perfeccionamiento Profesional.

\section{ENCONTRO AMAZÔNICO DE AGRÁRIAS - ENAAG}

Durante o congresso nacional de estudantes de agronomia (CONEA) de 2007, a interação entre alunos da UFRA (Belém) e UFPA (Marabá), foi o pontapé inicial para conversas sobre os eventos acadêmicos na região norte do Brasil. O amigo Ronei Silva (UFPA Marabá), comentava sobre o Encontro Regional de Agrárias (ERA) que já era organizado pelos alunos de Agronomia da UFPA Marabá e ressaltou que a inspiração foi a Semana de Integração das Ciências Agrárias (SICA) realizada pelos alunos de Agronomia da UFPA Altamira. O engajamento, importância e contribuição destas equipes de trabalho relatados pelo amigo, criaram o questionamento:

\section{E a UFRA quando terá algo semelhante?}

No ano seguinte ocentro acadêmico de agronomia (Ceagro), que passou a incentivar a participação dos alunos da UFRA em eventos acadêmicos regionais, viabilizando a participação de 12 alunos (Agronomia e Medicina Veterinária) no ERA 2008 em Marabá, foi a primeira vez que um grupo daUFRAparticipou do evento. Durante a mesa de encerramento do evento, com todos os coordenadores destes eventos, o Ronei Silva expôs o questionamento outrora levantado: 
Eu não entendo como a UFRA, uma universidade grande e localizada na capital, não consegue organizar um evento nos moldes do que nós fazemos aqui em Marabá, Altamira e Imperatriz.

Após isso, imediatamente eu e os demais alunos da UFRA no evento, profundamente incomodados e nos sentindo desafiados reunimos de forma quase que intuitiva e após alguns minutos de conversa decidimos por desenvolver a ideia de organização de um evento acadêmico no ano seguinte. A proposta havia nascido e o desafio estava lançado, naquele momento: Deyvison Medrado Gonçalves (Agronomia); Diego Vianna (Agronomia); Ellison Oliveira (Agronomia); Gleycianne dos Anjos (Medicina Veterinária); Paulo Adam Matos (Agronomia); Suellen Araújo (Agronomia); Tiéure Ribeiro (Agronomia) eram os presentes e foram os precursores do que viria a se tornar o Encontro Amazônico de Agrárias.

\section{PRIMEIRO EVENTO}

A equipe de trabalho começou a desenvolver atividades fundamentais como: definição do nome, criação de logo e o mais importante, as diretrizes e o que desejávamos alcançar com o evento, com isso criamos o primeiro esboço do que viria a ser o ENAAG. No início as reuniões eram bem difíceis, pois não tínhamos noção alguma de como organizar um evento deste porte e com a responsabilidade toda em nossas mãos. Neste momento o recém transferido da UFPA Altamira, Diego Nascimento passou a transmitir a experiência adquirida na organização da SICA do ano anterior e por insistência dele a coordenação decidiu participar dos outros dois eventos que ainda iriam acontecer naquele ano.

No entanto, a prioridade era que toda a coordenação pudesse ir a Semana de Integração das Ciências Agrárias em novembro. Desta forma apenas eu (Deyvison Medrado) e Diego Nascimento participamos (com recursos próprios) da Semana de Agronomia na UEMA Imperatriz, com o intuito de observar a dinâmica do evento e absorver o máximo de experiência com os organizadores. Porém, ao final do evento nos foi aberto um espaço para falarmos um pouco da nossa proposta de construir um ENAAG em 2009. Então, por conta própria informamos que o evento iria acontecer, inclusive estipulamos uma data prevista, e iniciamos a divulgação. Com certeza a partir desse momento o ENAAG começou a sair das 
reuniões esporádicas na biblioteca e do papel, de fato. Tanto, que este é o momento que nós consideramos de alta importância pela pressão que foi criada para que tudo fosse construído realmente.

Alguns meses depois nós já estávamos bem mais adiantados, principalmente pelo fato de já existir uma conversa firmada com o professor Sérgio Gusmão, que naquela ocasião foi nosso tutor e a pessoa a dar o respaldo ao primeiro projeto assim como a pró reitoria de extensão, então comandada pelo Prof. Lauro Itó, outro grande incentivador do evento. Estes dois professores foram as primeiras pessoas na instituição a acreditar na ideia e principalmente na palavra daqueles alunos de graduação.

Mas ainda restava um ponto de observação antes de o ENAAG existir, a viagem até Altamira, nos lançamos na Transamazônica e encaramos o desafio. Essa foi uma das semanas mais produtivas para a coordenação do ENAAG, pudemos enxergar muitas coisas boas para aproveitarmos, assim como alguns pontos a melhorar. Naquele momento tínhamos todos os elementos necessários para nosso crescimento. Fizemos a divulgação do evento durante a cerimônia de encerramento da SICA, já anunciando datas e o tema do evento. Eis que então o secretário de agricultura do Estado se coloca a disposição para ajudar no que fosse possível com o ENAAG. Pronto, tínhamos conseguido um patrocinador. A partir desse momento nosso trabalho se intensificou de vez, com cada vez mais reuniões da coordenação, com possíveis patrocinadores, fornecedores e gestores da Universidade.

O nome Encontro Amazônico de Agrárias, foi uma sugestão do Ellison Oliveira e apoiada por unanimidade, nosso objetivoera que o nome representasse desde o início transposição das fronteiras da UFRA e do Estado do Pará. Objetivando atender essa expectativa, criamos nosso primeiro diferencial dos demais eventos inspiradores, a coordenação deveria ser multi cursos. Precisávamos de pessoas de outras áreas, por motivos simples, entender o que o estudante dos cursos da UFRA e da Amazônia gostariam de ter em um evento acadêmico e atender a demanda da própria UFRA em relação a cursos de curta duração, que naquela época eram apenas oferecidos na semana do PIBIC com cargas horárias mínimas, disso surgiu nosso segundo diferencial, cursos de curta duração com carga horária de 20h, exatamente o mínimo exigido nos editais dos programas de pósgraduação da UFRA para obtenção de pontuação curricular. Além disso, permitir a participação de todos com taxas de inscrição simbólicas. Os valores arrecadados eram o 
suficiente (nem sempre) para manter o evento funcionando e poder ofertar espaços com qualidade para os participantes sem que houvesse segregação e todos pudessem ter acesso ao evento que estávamos criando.

Em resumo, o ENAAG em 2009 aconteceu e foi um sucesso, talvez até maior do que esperávamos, recebemos um feedback muito positivo dos participantes e palestrantes. Ainda assim haviam muitos pontos a melhorar para o ano seguinte, que para nós era o maior desafio, manter o sucesso e o crescimento ao longo dos anos, mas a dúvida era como fazer isso? Não sabíamos, mas conseguimos. Sempre buscamos ouvir todas as sugestões possíveis e principalmente as críticas, para não repetirmos erros. O segundo ENAAG foi bem maior que o primeiro, teve adição de mais alguns alunos a coordenação e houve um crescimento no número de participantes.

No ano de 2011 decidimos que era hora de evoluir e incorporar publicação de resumos expandidos e apresentação de trabalhos. Para isso criamos uma comissão científica que foi responsável pela elaboração do edital, modelos de resumo e o desenvolvimento dos anais do evento, para isso convidamos dois professores da instituição para, junto com os alunos, conduzirem o registro junto a biblioteca da UFRA e a Nacional para obtenção do ISBN para a publicação. Convidamos a professora Iris Lettiere na época, coordenadora do curso de Agronomia e o professor Raimundo Nelson Souza da Silva então pró-reitor de extensão. Dentro da comissão científica, como eu (Deyvison) era o mais antigo acabei sendo indicado para junto com os professores liderar a criação da publicação dos resumos expandidos do evento. Para nossa surpresa a submissão de trabalhos ultrapassou todas as expectativas em questões de números e qualidade, além de se tornarem um motor para o crescimento do número de inscrições no evento.

Já na quarta edição as inovações foram as redes sociais, fortalecemos nossos perfis e avançamos na criação de um site. A ideia do site já vinha sendo debatida, mas até então não havia decolado, por nossa conta decidimos que iríamos aprender e desenvolver um site, por mais simples que fosse, mas que deveria ter um visual agradável e que fosse fácil para os usuários e para os futuros coordenadores. Com o site em funcionamento, conseguimos gerir as inscrições, tínhamos uma linha de contato com os interessados no evento e o melhor daquele ano: a disponibilização on-line de todos os trabalhos já publicados nas edições anteriores e o que potencialmente nos eliminava a necessidade de produção de cd's. Era o 
que precisamos para reforçar o apelo ambiental do evento, que desde a primeira edição já existia, com a redução do uso de copos descartáveis e distribuição de copos junto ao kit do participante.

Diante de tanto investimento na divulgação digital, na quarta edição do evento tivemos inscrições de outros estados da região amazônica como: Amazonas e Mato Grosso, que por sinal chegou a UFRA com cerca de 80 participantes, isso simbolizava que nós havíamos atingido um dos nossos objetivos principais, extrapolar as fronteiras do estado do Pará. Era hora da primeira grande renovação, os novos coordenadores estavam prontos para assumir a responsabilidade.

O principal objetivo da nossa ideia era transformar o ENAAG em um evento oficial da UFRA, com apoio, datas no calendário acadêmico e principalmente reconhecimento dos gestores. Não foi nem um pouco fácil, tínhamos que provar a cada ano que o evento era permanente e não servia para arrecadação financeira de comissões de formatura, o que até aquele ano era bem comum de acontecer. Além disso, queríamos construir conhecimentos que não eram possíveis de adquirir nas salas de aula, coisas como organização, trabalho em equipe, liderança, oratória, gestão financeira, tomadas de decisão rápidas e sob pressão.

A transição da coordenação que outrora havia iniciado esse ideal deu-se de forma inusitada, pois foi uma grande responsabilidade repassada para os novos coordenadores que agora seriam os coordenadores referência para que tocassem a coordenação e o evento acontecesse, destacamos que edições com o passar dos anos estavam tornando-se melhores e esse desafio de mudança total de coordenação não poderia refletir no evento, ele deveria seguir o fluxo e continuar a crescer dentro e fora da instituição.

A coordenação que foi formada a partir dessa mudança compreendeu o papel do ENAAG para com a universidade e respondeu o que era um questionamento antes feito: será que teríamos ENAAG no próximo ano? A resposta que conseguiram depois de muitas incertezas foi positiva e sim ele aconteceu! E foi um número recorde de inscritos, diga-se de passagem.

Os ministrantes que vinham de outras instituições como a Professora Thereza Camello (UERJ) abraçaram a coordenação e o evento como se fossemos seus "filhos" ficou maravilhada com nossa força de vontade e determinação para realização do evento, tanto 
que buscou contribuir e unir laços entre as duas instituições oportunizando aos melhores trabalhos científicos apresentados poderem ser publicados no site da revista da sua universidade. Outro professor, Ivan Crespo (UFPR), que achou extraordinário ter um evento com tamanha magnitude e ser coordenado por discentes, que além do compromisso de planejar e executar o evento, tem afazeres para com a formação, estágios, trabalhos e ainda sim se dedicar para que um evento desse porte aconteça. Ele chegou a comparar que ainda não havia visto, por onde andou evento igual ao nosso, gostou tanto que, em uma das edições fez o lançamento de um livro de sua autoria com título "Sistemas Agroflorestais, conceitos e métodos".

O ENAAG criou laços com empresas juniores para estas contribuírem com cursos e palestras além da divulgação das empresas durante o evento, assim como com os grupos da universidade (CA's PETS, IARA) percebendo sobre importância do ENAAG, passaram a nos incluir e defender quando foi preciso, ser incluído datas de calendários acadêmicos expondo que os cursos ofertados no ENAAG eram algo que já estava enraizado como sendo algo da UFRA.

A Coordenação com o passar das edições foi se modificando e com isso buscando melhorar a arte do ENAAG, Murillo Azevedo tendo conhecimento sobre edição de imagens plotou a vitória régia que fora escolhida para ser símbolo do ENAAG, melhorando a sua visualização para divulgação e serigrafia. Palestras simultâneas foram pensadas como forma de abranger os mais variados ramos das agrárias, que possibilitou ser organizada por eixos (produção agrícola e florestal, saúde e produção animal e recursos ambientais e pesqueiros).

O comitê 2015 (Bryza Sanco; Brenda Reis; Luana Aires; Maryelle Sousa; Lais Carvalho) questionou por que não era possível inovar na forma de apresentações dos trabalhos científicos e depois de muito pensar sobre como seria feita, eis que surgiu a ideia de que os dez melhores trabalhos científicos iriam ser convidados a apresentação de forma oral e que haveria duas premiações distintas, uma para os dois melhores trabalhos apresentados em pôster e outros dois melhores em apresentação oral. A busca em formalizar os meios de divulgação via eletrônica foram mudadas, plataformas de site, e-mails de comunicação pelas comissões foram reformulados e estão sendo passadas adiante, como forma de preservar as informações contidas das edições passadas para nortear e servir de apoio para duvidas a que venham surgir ao longo do processo de construção. 
Com passar das edições e da chegada de novas pessoas que ingressavam na coordenação algumas ideias foram reformuladas. A ação social que durante as cinco primeiras edições foi arrecadação de alimentos, que era de acordo com inscrição no curso mais um de quilo alimento não perecível para doação a instituições afins, e foi mudada para ser um quilo de ração para cão/gato no qual era referente a inscrição em palestras ofertadas no evento e a arrecadação era doada a instituições afins.

Outra mudança ocorrida foi a do perfil de coordenador que ingressa no ENAAG, os antigos, daquela geração antes da primeira grande mudança, você sentava, ouvia, acompanhava, se espelhava neles por terem vivenciado tudo que estava sendo construído e nisso foi percebida mudança.

Com o passar dos anos e das edições do evento, as pessoas que iam ingressando na Universidade, consequentemente na coordenação, foram mudando, e também as indicações dos coordenadores apresentaram essa modificação. Num primeiro momento, até a quinta edição, as indicações eram por perfis de alunos proativos e que se encaixavam em alguma comissão das formadas na coordenação. Com passar dos anos e repercussão do reconhecimento e do "status" que os alunos que eram da coordenação acabaram adquirindo, isso foi crucial para que algumas pessoas quisessem fazer parte de um grupo de alunos com tal reconhecimento, embora não soubesse como funcionavam os bastidores para formulação e execução do evento.

Exercer um controle rigoroso nos processos de recrutamento de novos integrantes como apoio e/ou coordenação é algo plenamente possível, basta seguir uma agenda comprometida com escolhas que atendam as necessidades e desenvolver mecanismos de seleção que permitam aos participantes valorizarem a vaga pretendida, isso fortalecerá a meritocracia como critério de inserção de novos colaboradores na equipe. Se os selecionados não tiverem as exigências mínimas para a função, certamente o projeto enfrentará percalços a curto, médio ou longo prazo, levando a resultados decepcionantes. Não podemos esquecer que somos multiplicadores de uma causa que ultrapassa os limites territoriais da UFRA, por isso princípios éticos e de respeito entre os integrantes é fundamental para o processo interativo do grupo, como disse Sun Tzu, "a água sem queda não pode correr; tropas malconduzidas não podem vencer" (dito por Daniel Santos, ENAAG 2015). 
De fato, em alguns momentos foi observada uma falta de experiência quanto ao sentido do ENAAG, chegando a comparar o evento a outros que ocorriam com preços mais elevados, a tal ponto que foi questionado o porquê do ENAAG ofertar cursos tão baratos? (visto que outros eventos da própria instituição cobravam cursos iguais e com valores mais altos). Porque não seria a hora de aumentar o valor da inscrição para dar ao evento tal elevação de importância? Por decisão da maioria da coordenação que já estava composta até o momento esse questionamento, foi moldado e reformulado. Restava ver como o aluno, que já estava acostumado com preço em conta do evento iria reagir a isso, assim como para alguns da coordenação passada isso foi um grande pulo no escuro, para os participantes foi motivo de muita reclamação e questionamento.

Um acontecimento, a partir da sétima edição, foi crucial para que a taxa de inscrição no evento sofresse um aumento de preço maior do que o de costume. Desde que foi criado o ENAAG em termos financeiros foi administrado pelos alunos. Devido acontecidos na UFRA, os projetos de extensão vinculados a PROEX deveriam estar financeiramente vinculados a Fundação de apoio à pesquisa, ensino e extensão - FUNPEA, o Pro reitor de Extensão Djacir Ribeiro expôs a coordenação, pois caso houvesse alguma averiguação sobre as arrecadações do evento, estas teriam como ser comprovadas. Com essa nova forma de vinculo financeiro o ENAAG iria ter que arcar com uma taxa administrativa para esta fundação o que nas edições passadas nunca havia ocorrido. Os valores arrecadados nas inscrições dos cursos são para custear o evento como um todo, ter uma taxa de gasto a mais pesou sobre a taxa de inscrição que teve que acrescentar para que fosse possível executar o evento e pagar a taxa financeira.

Com isso as formas de compra e pagamentos dos materiais mudaram de formato, quando era feito pelos coordenadores sem vínculo com a fundação a busca por preços mais baixos era possível visto que obtínhamos para nosso controle financeiro notas simples. Com a fundação estando como detentora do recurso financeiro a coordenação deveria apresentar três cotações e a mais barata seria comprada, essa transição financeira de operação limitou a coordenação em aspectos de facilidade para utilização do recurso além de comprar materiais com preços mais elevados devido emissão de nota fiscal. 
Bom, agora em termos de palestras e cursos de curta duração, sim a maioria teve êxitos e aumentaram mais ainda a credibilidade do ENAAG em ofertar cursos com preços em conta e com aulas práticas/teóricas que foram pelos participantes ditas como proveitosas, tanto que hoje as inscrições são esperadas pelos participantes que todo ano vem por medo dos cursos esgotarem rápido demais, o que de fato ocorre em alguns cursos que são bem concorridos. As temáticas abordadas e organizações para cada edição ficaram tão bem mencionadas que professores e pesquisadores veem hoje se disponibilizar para contribuir no ENAAG na forma de palestra ou minicurso, algo que num momento anterior nós que íamos ao encontro deles, ver o evento ter essa seriedade é gratificante.

Daqui em diante haveria mais uma transição de coordenação, para os novos coordenadores tomarem a frente, assim como foi feito na vez passada, contudo muitos não quiseram puxar tal responsabilidade para si ou visaram outros caminhos a seguir. Paramos e nos perguntamos, o que será que ocasionou isso?Qual o desafio em construir algo a partir do que vivenciou? Ou que aquilo não era o mais importante no momento, talvez outros caminhos os tenham chamado e ir sozinho nessa estrada não parecia convidativo, afinal teríamosque montar a própria caminhada!

Após todas as edições, das quais a coordenação lutou para que o evento fosse reconhecido pela Universidade como sendo um evento da UFRA, na consolidação da nona edição e decisão da data na reunião de conselheiros da UFRA (CONSUN) o ENAAG que ate a oitava edição teve que demonstrar sua importância e relevância do por que "parar" a UFRA por uma semana para oportunizar aos alunos um evento que venha somar aos conhecimentos adquiridos na universidade. Os coordenadores da nona edição tiveram apoio unânime dos conselheiros para realização do evento, mérito que foi alcançado após anos de luta e de muita insistência.

A coordenação da nona edição por ter passado por um processo de avaliação minucioso e seletivo, obteve uma resposta positiva nesse início, alguns engajaram-se e outros foram esforçando-se para corresponder às expectativas.

A coordenação decidiu arcar com a compra de um site e uma nova logomarca, substituindo o anterior, ao ENAAG que firmou algo que será perpetuado. 

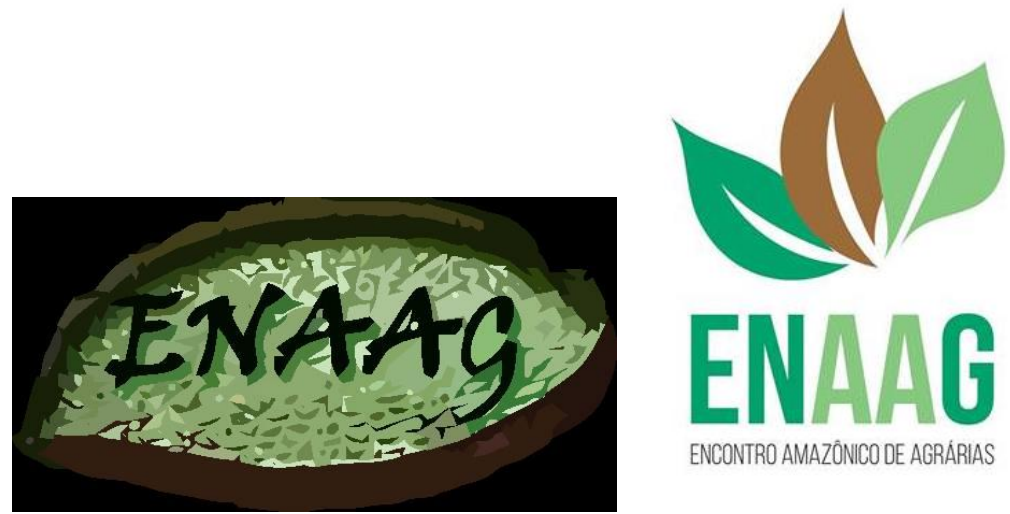

Participar da coordenação do ENAAG por quatro ou cinco anos, nos ajudou a desenvolver essas habilidades e acredito que meus amigos também conseguiram o que nos deixa extremamente orgulhosos por termos sido parte importante no desenvolvimento pessoal e profissional de cada um deles.

O conhecimento e experiência adquirida ao longo dos anos como coordenadora ENAAG oportunizou nos moldarmos como profissional e como pessoa. Conhecemos pessoas maravilhosas que nos encorajavam diante da dimensão que o evento chegou e ainda tem muito a crescer. Consideramos um ensinamento a mais que só vivenciando para saber como é, e nenhum ENAAG é igual ao outro, cada evento é único.

Em 2018 o ENAAG irá completar 10 anos de existência e aparentemente com o mesmo fôlego e empolgação daqueles alunos lá em 2008. Acho que sim, deixamos nosso maior legado na UFRA, era isso que desejávamos fazer com que simples alunos de graduação pudessem exercer sua criatividade e pensar fora da caixa, fora dos muros da UFRA.

Talvez algo muito maior que isso, acreditamos que após a criação do ENAAG conseguimos despertar na comunidade UFRAniana o interesse por esse assunto, a exemplo de outras universidades onde ao longo do ano há diversos eventos de diferentes portes realizados por alunos de graduação e pós, por grupos de pesquisa e entre outros interessados em contribuir. O que queremos? Que todos cresçam.

Conseguimos criar, contribuir, aprender e estimular outros a continuarem investindo no projeto do ENAAG, feito por alunos e para alunos. 
Agradecemos a todas as coordenações que lutaram e mantêm vivo o espírito do ENAAG.

\section{Coordenações:}

ENAAG 2009: Deyvison Diego Vianna, Diego Nascimento, Suellen Araujo, Gleyceanne Anjos, Tieure Ribeiro, Lucio Mendes, Larissa Soares e Lorena Lira.

ENAAG 2010: Leticia Sales, Sara Baima, Michelle Melo, Deyvison Medrado, Diego Vianna, Diego Nascimento, Tieure Ribeiro, Lucio Mendes, Larissa Soares e Lorena Lira.

ENAAG 2011: Diego Nascimento, Leticia Sales, AlanNahon, Larissa Soares, Tieure Ribeiro, Andreia Erdmann, Pedro Leal, Igor Barros, Sara Baima, Felipe Castilho, Deyvison Medrado, Edwin Assuncao, Lucio Mendes, Giulia Venturieri, Susana Silva e Camila Maciel.

ENAAG 2012: Deyvison Medrado, Alan Edir Nahon, Giulia Rury Venturieri, Camila Maciel de Souza, Susana Silva, Edwin Almeida, Renato, Adria Azevedo, André Oliveira, Izabel Cristina, Igor Barros, Bruna Oliveira, Ian Kiyoshi, Avner Gaspar, Rafael Lira, Juliana Rodrigues, Rayssa Braga, Anna Silva.

ENAAG 2013: Bruna Oliveira, Marina Passos, Renato Rodrigues, Rayssa Braga, Anna Silva, Lais Carvalho, Rhannaldy Benicio, Daniel Silva, Thamara Cozzi, Adriana Neves, Murillo Azevedo, Caio Costa, Mayara Pereira, Nilson Almeida, Savio Guerreiro, Ian Kiyoshi Yamamoto, Bryza Sanco.

ENAAG 2014: Duane Azevedo, Mayara Pereira, Matheus Guedes, Daniel Silva, Jessika Magalhaes, Bruna Oliveira, Aline Ceci, Daniel Santos, Raissa Rafaela, Bryza Sanco, Lais Carvalho, Luciana, Luiz, Bruna Figueiredo, Andre Monteiro, Jennifer, Hilda Azevedo.

ENAAG 2015: Marina Passos, Maryelle Machado, Daniel Santos, Raissa Rafaela, Bruna Oliveira, Hilda Azevedo, Jeanderson Viana, Iago Rodrigues, Lorena Maués, Paula Marques, Leticia Felix, Lais Carvalho, Andrezza Farias, Adriano Cardoso, Guilherme Mendes, Andre Monteiro, Luana Aires, Ricarla Viana,

ENAAG 2016: Ricarla Viana, Bruna Oliveira, Amanda Lobato, Eliene Araujo, Helen Kamile, Maura Brochado, Italo Leal, Iago Rodrigues, Guilherme Mendes, Érika De Paula Mercês, Daniel Silva, Gustavo Seribeli Furmigare, Leonardo Brandão, Crislene Monteiro, 
Clayciane Nascimento, Katiane Sousa, Dayseanne Alencar, Pâmela Bahia, Manoela Torres, Claudia Carvalho, Adriano Cardoso, Leticia Felix, Brenda Reis, Luana Aires, Lorena Maués.

ENAAG 2017: Amanda Lobato, Ricarla Viana, Igor Borges Mesquita, Hélio Aguiar, Alba Rocha, Tainara Nunes, Tinayra Teyller, Wenderson Ferreira, Nicolas Rodrigues, Marcus Santiago, Louise Regateiro, Wenderson Hollanda, Amanda Mendonça, Helene Castro, Tales Lopes, Bruna Oliveira, Milana Carla dos Santos Mendes, Raul Coimbra Miranda. 